\title{
Software Defined Radio-based Mixed Signal Detection Laboratories for En- hancing Undergraduate Communication and Networking Curricula
}

\author{
Dr. Zhiqiang Wu, Wright State University
}

Dr. Zhiqiang Wu received his BS from Beijing University of Posts and Telecommunications in 1993, MS from Peking University in 1996, and PhD from Colorado State University in 2002, all in electrical engineering. He has worked at West Virginia University Institute of Technology as assistant professor from 2003 to 2005. He joined Wright State University in 2005 and currently serves as full professor. Dr. $\mathrm{Wu}$ is the author of national CDMA network management standard of China. He also co-authored one of the first books on multi-carrier transmission for wireless communication. He has published more than 100 papers in journals and conferences. He has served as Chair of Acoustic Communication Interest Group of IEEE Technical Committee on Multimedia Communications. His research has been supported by the National Science Foundation, Air Force Office of Scientific Research, Air Force Research Laboratory, Office of Naval Research, and NASA. His work on software defined radio implementation of cognitive radio won the Best Demo Award at IEEE Globecom 2010.

Prof. Bin Wang, Wright State University

Prof. Bin Wang earned his Ph.D. from the Ohio State University in 2000. He joined the Wright State University in September 2000, where he is currently full professor of computer science and engineering. His research interests include optical networks, real-time computing, mobile and wireless networks, cognitive radio networks, trust and information security, and semantic web. He is a recipient of the US Department of Energy Career Award. His research has been supported by US Department of Energy, National Science Foundation, Air Force Office of Scientific Research, Air Force Research Laboratories, Ohio Supercomputer Center, and the State of Ohio.

\section{Prof. Chi-Hao Cheng, Miami University}

Dr. Chi-Hao Cheng received the B.S. degree in control engineering from National Chiao Tung University, Taiwan in 1991, and the M.S. and Ph.D. degrees from The University of Texas at Austin in 1996 and 1998 respectively, both in Electrical and Computer Engineering. He is currently a professor in the Department of Electrical and Computer Engineering at Miami University, Ohio. His primary professional interests lie in signal processing algorithm development and its applications in numerous communications system and component development including wireless and optical communications systems. He is co-inventors of three US patents.

\section{Dr. Deng Cao, Central State University}

Dr. Deng Cao received his Ph.D in Computer Science from West Virginia University in 2013. He also earned two master degrees in Statistics and Physics from West Virginia University. Dr. Cao joined Central State University in 2013 and currently serves as an assistant professor in the department of Mathematics and Computer Science. His research interests include computer vision, pattern recognition, machine learning, and advanced biometrics. .

\section{Dr. Zhiping Zhang, Wright State University}

Zhiping Zhang received his B.S. degree in electrical engineering from Nankai University, Tianjin, China, in 2001, M.S. and Ph.D. degrees in intelligence science from Peking University, Beijing, China, in 2004 and 2011 respectively. From 2011 to 2013, he was a Postdoctoral Research Fellow at the Department of Computer Science and Technology, Peking University. Since 2013, he has served as a research faculty member and co-director of Broadband, Mobile and Wireless Networking Laboratory at the Department of Electrical Engineering of Wright State University. 


\title{
Software Defined Radio based Mixed Signal Detection Laboratories for Enhancing Undergraduate Communication and Networking Curricula
}

\begin{abstract}
Communication and networking courses, especially wireless communication and networking courses, have become more and more important in many disciplines such as Electrical Engineering, Computer Science, and Computer Engineering. Due to costly hardware needed for communication and networking teaching laboratories, many of these courses are taught without a laboratory. In the rare cases of existing labs, such hardware based teaching labs lack the flexibility to evolve over time and adapt to different environments.
\end{abstract}

Supported by a NSF TUES type II project, we have developed a series of software defined radio (SDR) based mixed signal detection laboratories for enhancing undergraduate communication and networking curricula. In our previous NSF funded CCLI project "Evolvable wireless laboratory design and implementation for enhancing undergraduate wireless engineering education", we have developed and demonstrated the first nationwide example of evolvable SDR based laboratories for three existing undergraduate courses.

In this project, we are developing new lab components that can be adopted by multiple courses ranging from freshman year introductory course to senior year capstone design projects. Specifically, in this paper, we report the development of a SDR based mixed radio frequency signal detection platform with a graphical user interface (GUI). This user-friendly GUI will allow students to adjust RF parameters such as carrier frequency, symbol rate, pulse shaping filter, etc., and mix multiple RF signals together. Additionally, students are able to observe the transmitted signal in both time and frequency at both transmitter and receiver. At receiver side, the SDR based platform also provides students the functionality of performing RF signal detection via different detection methods including energy based detection, waveform based detection, and cyclostationary analysis based detection. It is shown that by exploiting sophisticated signal processing techniques such as cyclostationary analysis, mixed RF signal components can be detected and identified.

Through collaboration among the three participating institutions (Wright State University, Miami University (a mostly undergraduate serving institution), and Central State University (an HBCU)), based platform will be integrated in undergraduate curricula of all three institutions. 


\section{Introduction}

Need and Motivation: In this paper, we report a series of software defined radio based mixed signal detection laboratories to give students hands-on experiences with radio frequency signal detection and estimation. Signal detection and RF parameter estimation are very important topics for electrical engineering students, especially those who are specializing in communication and signal processing. However, most of the existing signal detection and estimation courses in universities are taught without a lab.

Electrical engineering and computer science are practical disciplines, and students learn by doing. Hands-on experience is an integral part of their learning. Students learn from equations and homework; they learn from computer simulations through Matlab and Labview; but nothing beats a real laboratory where they can get their hands dirty.

Traditionally, communication and networking laboratories are conducted using RF equipment such as radio frequency signal generators and spectrum analyzers, or custom-made/special laboratory equipment. Such equipment is always expensive and it is not feasible to purchase multiple sets of such equipment to accommodate an undergraduate teaching lab. To solve this problem, we have developed and demonstrated the first nationwide example of evolvable software defined radio (SDR) ${ }^{[1]-[7]}$ based communication and networking laboratories Supported by an NSF CCLI type I project, we developed an affordable SDR based platform and multiple experiments on it to serve three undergraduate communication and networking courses ${ }^{\text {[8][9]. }}$. Inspired by the success of the type I project, we have received support from an NSF TUES type II grant to extend and expand our effort to develop more teaching labs based on this SDR platform both vertically and horizontally. Specifically, we are designing and developing more lab modules to serve a wide range of courses from freshman year introduction courses to capstone senior design courses. Additionally, the three participating institutions of this project (Wright State University, Miami University (a mostly undergraduate serving institution), and Central State University (an HBCU)) are collaborating to deploy the lab modules developed at each individual institution across all campuses. We have presented our results from this project in previous ASEE conferences ${ }^{[10][11][12][13]}$.

In this paper, we present the newest result from our NSF TUES Type II project: a software defined radio (SDR) based implementation and demonstration of the cyclostationary analysis based mixed signal detection system. Specifically, employing USRP software defined radio platform and GNU radio software, we have developed a graphical user interface (GUI) controlled mixed RF signal generator and mixed RF signal detector. The mixed RF signal generator is capable of generating mixed RF signal with multiple individual signal components. The mixed RF signal detector is able to detect the existence of multiple individual signal components while traditional spectrum analysis fails to reveal the existence of mixed signals components.

\section{Mixed RF Signal Detection and Estimation}


In most existing work of signal detection and RF parameter estimation, it is often assumed that in any given spectrum band, there is only one single RF transmission. Fig. 1(a) shows this simplified spectrum scenario where multiple signals are located at different frequency bands, and a narrowband filter can be used to distinguish these narrowband transmissions. However, in an advanced communication system such as a cognitive radio network, multiple signals might significantly overlap in the spectrum. Fig. 1(b) illustrates the scenario where two narrowband transmissions with similar but different RF parameters mixed together. At the communication receiver, the resulting spectrum is illustrated in Fig. 1(c). It is clear that a conventional spectrum analysis is not capable of identifying that there are two signals mixed together here.

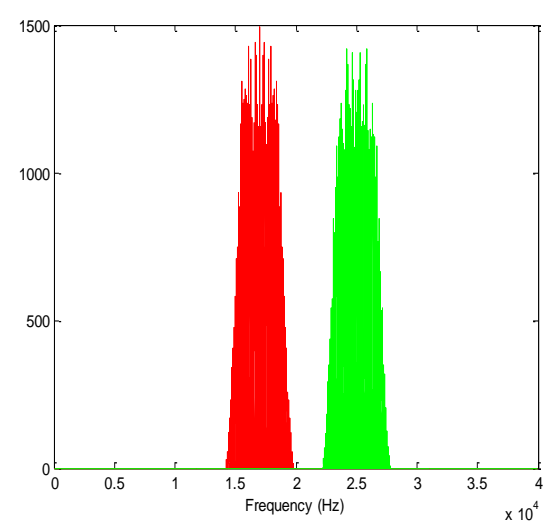

(a)

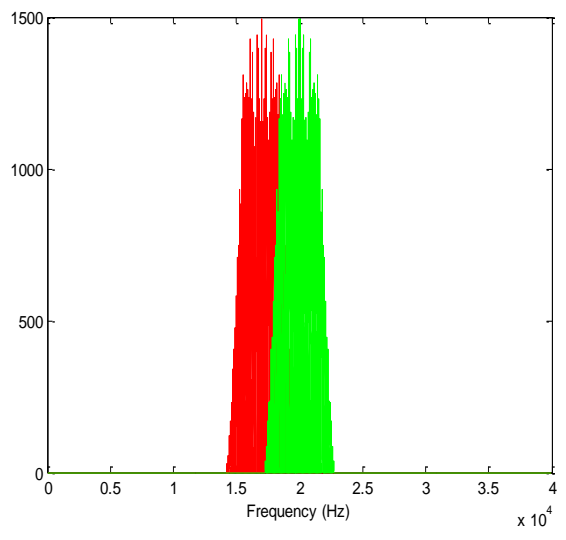

(b) 


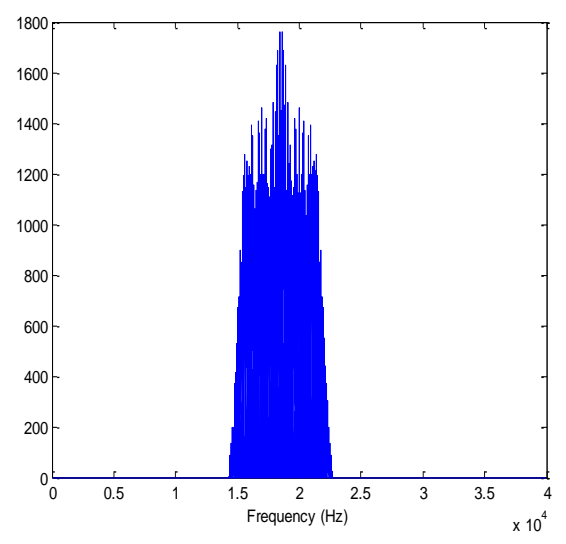

(c)

Fig. 1 Mixed Signals with Significant Spectral Overlap

The mixed signal scenario is not a big problem for conventional communication systems. However, to teach our undergraduate electrical engineering students with state-of-the-art communication technologies such as cognitive radio network, it is crucial to show them how to detect the existence of mixed signals, count the number of mixed signal components in each band, estimate the RF parameters of each signal component and identify their signal types.

\section{Software Defined Radio based Mixed RF Signal Detection Platform}

In our previous work, we demonstrated the feasibility of using second order cyclostationary analysis to detect and identify mixed RF signals through spectrum correlation function (SCF) and spectrum coherence function (SOF) ${ }^{[14][15][16][17]}$. By employing SCF and SOF features, we were able to find a stable threshold in our mixed signal detection algorithm to determine how many mixed RF signal components are present. We also evaluated the detection and parameter estimation performance under various channel conditions and signal mixture scenarios via numerical simulations.

Based on our previous work, we have developed a software defined radio based mixed RF signal generator and a software defined radio based mixed RF signal detector for enhancing undergraduate communication and networking curricula. Specifically, we have used the universal software radio peripheral (USRP) ${ }^{[6][7]}$ hardware by ETTUS and National Instruments because of its popularity in academia and its affordability. We have developed the platform on four different kinds of USRP boards: USRP1, USRP2, USRP N210, and USRP X300. We have also used arbitrary waveform generator, vector spectrum analyzer, wireless channel emulator and digital phosphor oscilloscope to validate and expedite the development of our lab modules.

Figure 2 illustrates the current set up of the software defined radio based mixed RF signal generator and mixed RF signal detector. As shown in Figure 2, on the left side, we have a GUI 
controlled SDR based mixed RF signal generator. On the right side, in Figure 2, shows the GUI controlled SDR based mixed RF signal detector. An Agilent vector spectrum analyzer shown in the middle of Figure 2 is used to validate the RF transmission and detection over the air.

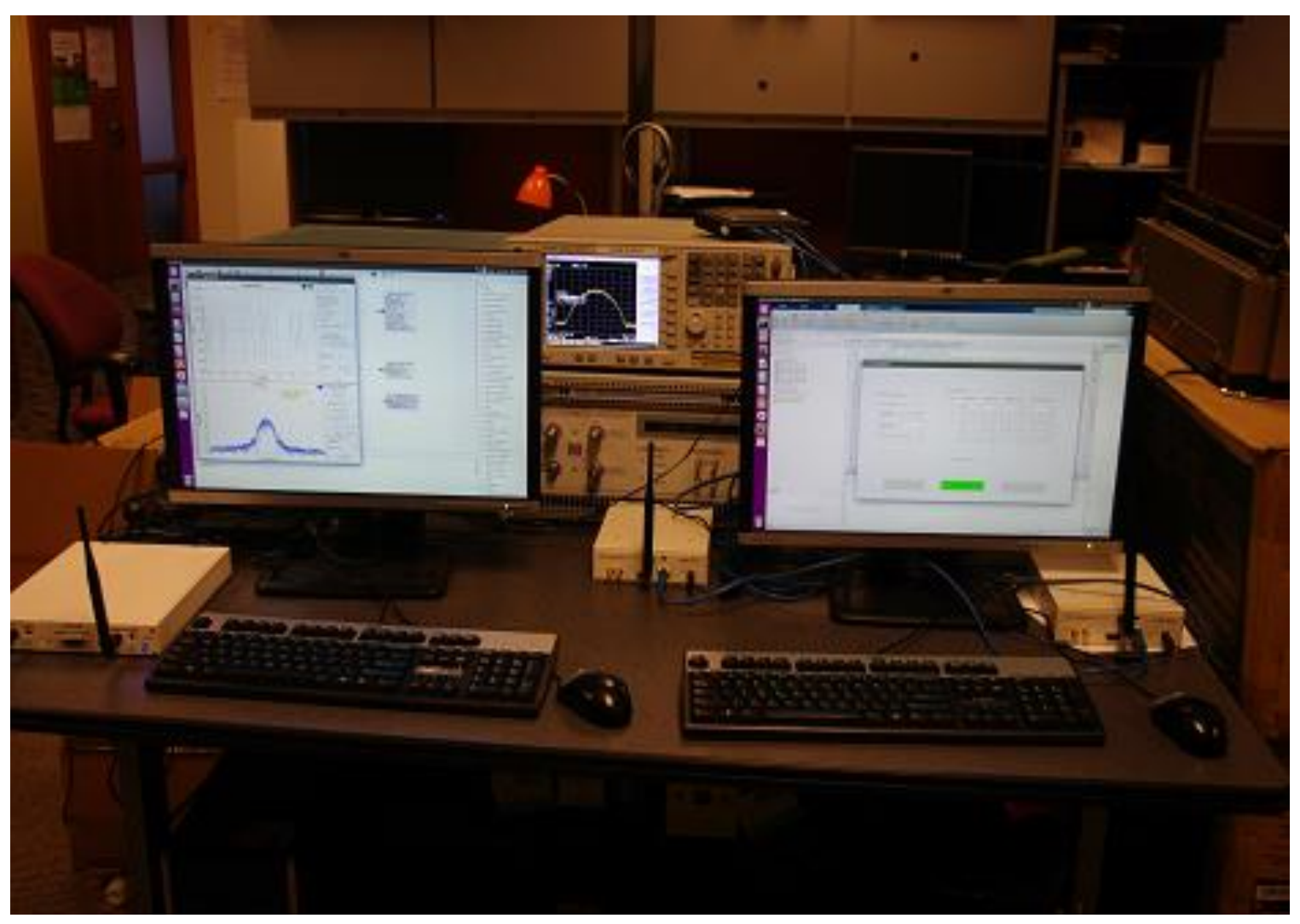

Fig. 2 SDR based Mixed Signal Generator and Mixed Signal Detector

\section{Laboratory Examples}

Many different laboratories can be designed and developed using this general RF signal detection platform. We have developed a graphical user interface (GUI) to control the SDR based mixed RF signal generator which allows users to easily adjust parameters of the desired mixed RF signal to be generated. Fig. 3 illustrates the GUI of the SDR based mixed RF signal generator. 

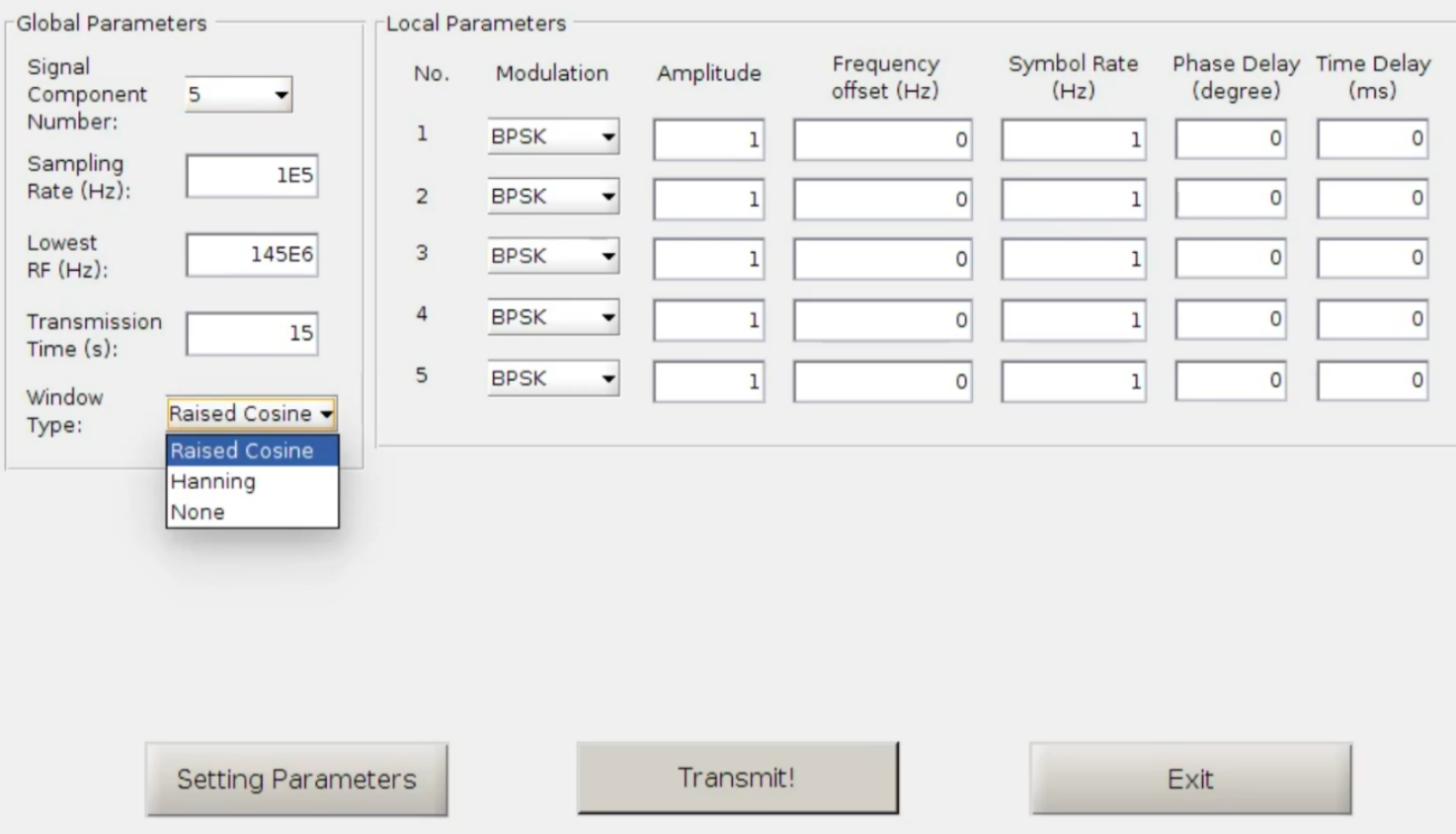

Fig. 3 GUI of SDR based Mixed RF Signal Generator

As shown in Figure 3, user can easily adjust the RF parameters to generate mixed (or non-mixed) RF signals. Specifically, the GUI provides a convenient interface to a user to control all RF parameters of the mixed RF signal generator. The functions designed in our system include:

(1) The number of individual signal components can be set;

(2) The sampling rate and transmission time can also be set;

(3) The shaping filter can be selected as rectangular function, Hanning function, or raised cosine function;

(4) Each individual signal component's modulation type can be chosen as BPSK, QPSK, 8PSK, or 16QAM;

(5) For each individual signal component, the amplitude, carrier frequency and symbol rate can all be set independently;

(6) Click the "Setting parameters" button, the mixed signal will be generated;

(7) Click the "Transmit!" button, the generated signal will be sent by the USRP SDR.

Figure 4 shows the GUI of the developed SDR based mixed RF signal detector. As shown in Figure 4, a button is pressed to obtain RF data from the USRP software defined radio boards for further processing. The user can easily adjust certain RF parameters including center frequency, sampling rate, frequency resolution, minimal cyclic frequency, maximum cyclic frequency, etc. After the data is obtained from USRP and also stored on the hard drive of the computer 
controlling the SDR, the mixed RF signal detector can perform time domain analysis, frequency domain analysis, and cyclostationary analysis.

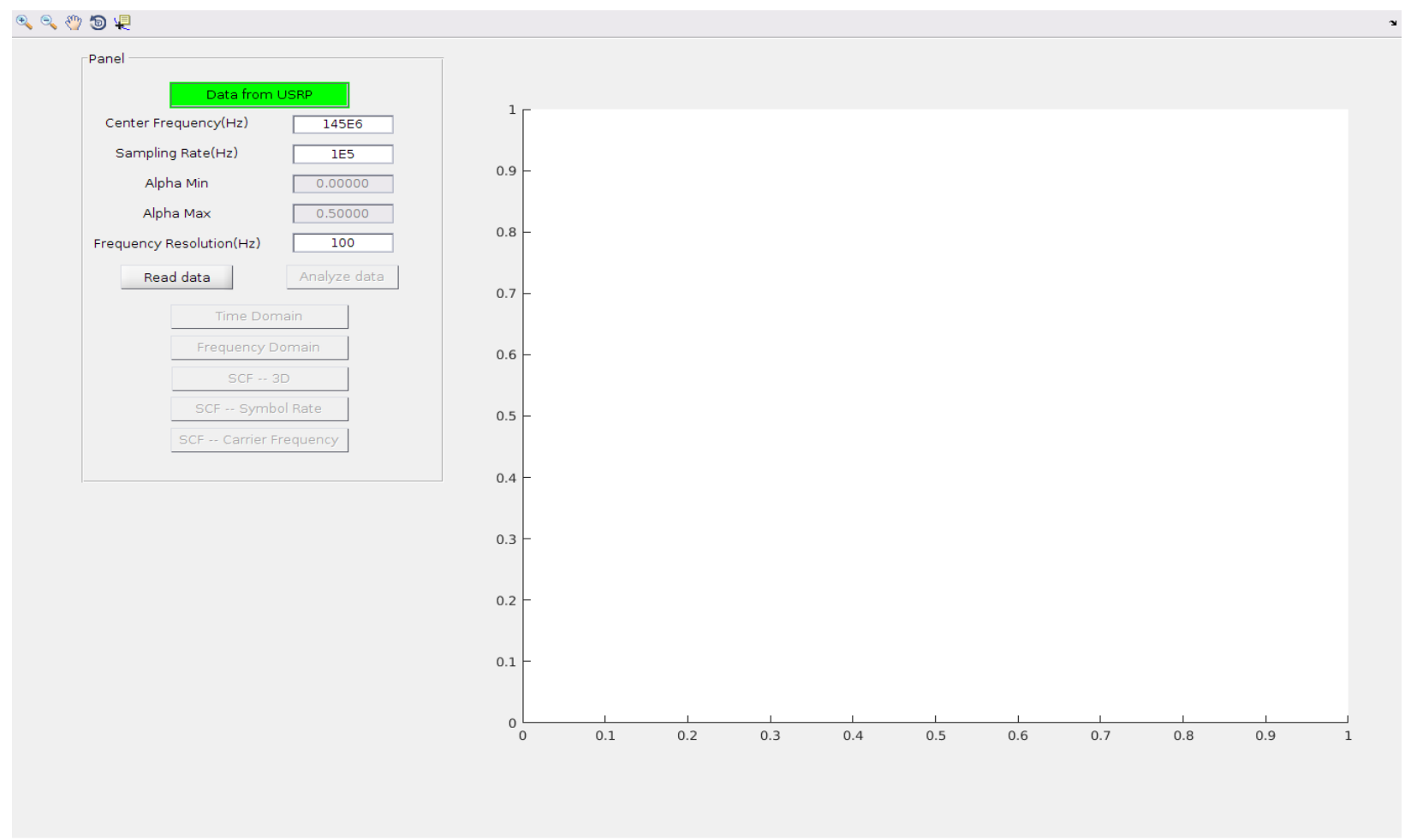

Fig. 4 GUI of SDR based mixed RF signal detector

Figure 5 shows the GUI of an example of a mixed RF signal generation of two individual RF signal components with significant spectrum overlap. As shown in Figure 5, we choose the carrier frequencies of two BPSK modulated individual signal components to be close to each other so they overlap significantly in spectrum. 


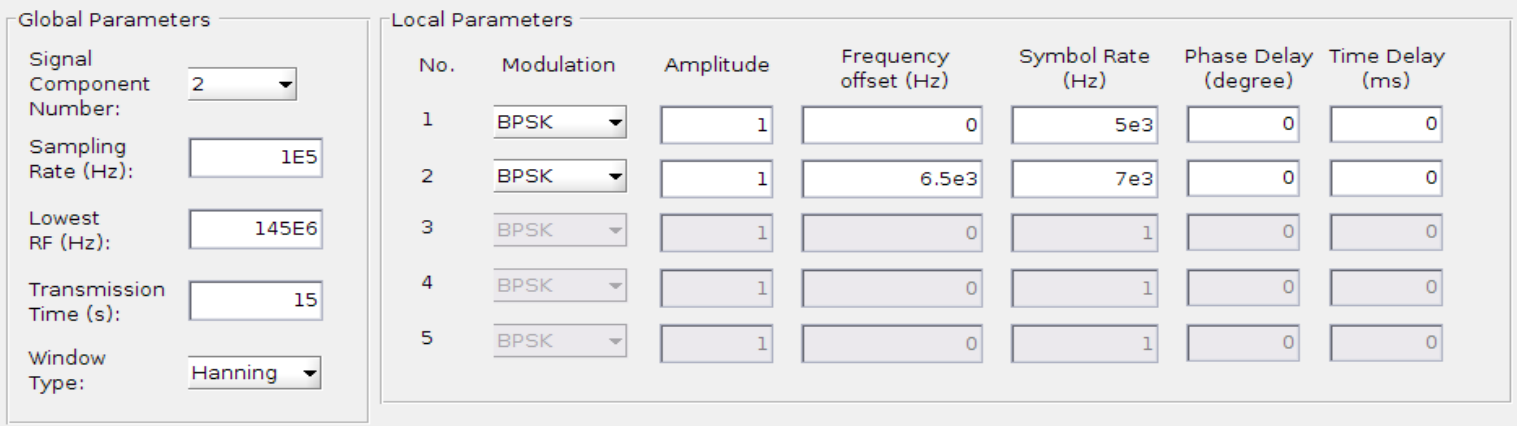

Transmitting. .

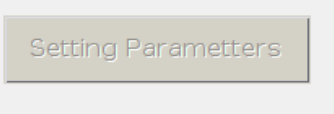

Transmitting

Fig. 5 Signal generation of signal with significant spectrum overlap

Figure 6 shows the time domain analysis result at the GUI of the SDR based mixed RF signal detector. Specifically, in-phase and quadrature components of the down-converted RF signal are displayed in the window on the right side of the GUI.

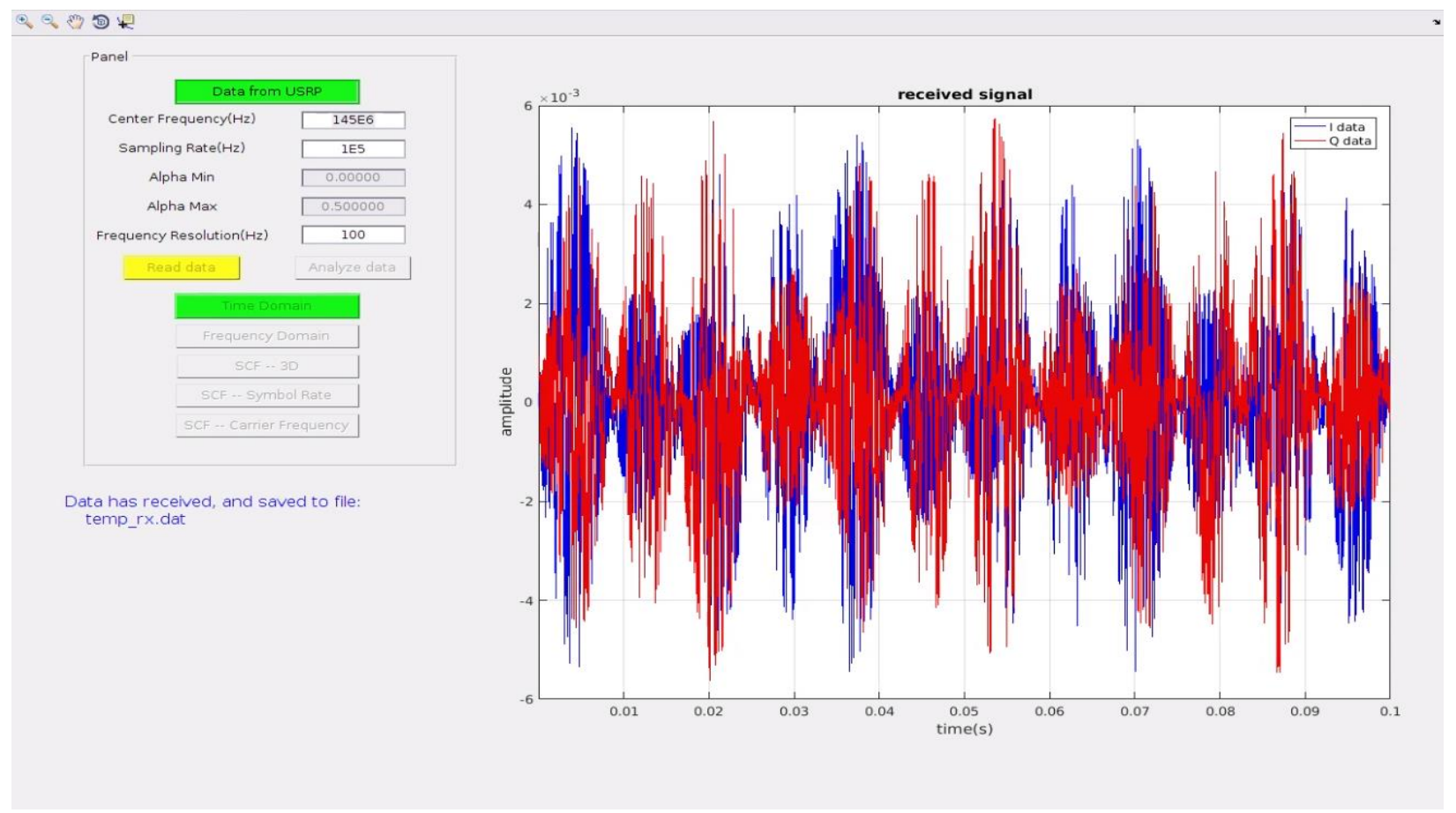

Fig. 6 Time domain analysis of signal with significant spectrum overlap 
Figure 7 shows the frequency domain analysis result at the GUI of the SDR based mixed RF signal detector. Specifically, fast Fourier transform (FFT) of the down-converted RF signal result is displayed in the window on the right side of the GUI. As seen in Figure 7, the two individual RF signal components are significantly overlapping in spectrum, therefore, the frequency domain analysis is incapable of detecting and distinguishing the two individual RF signal components.

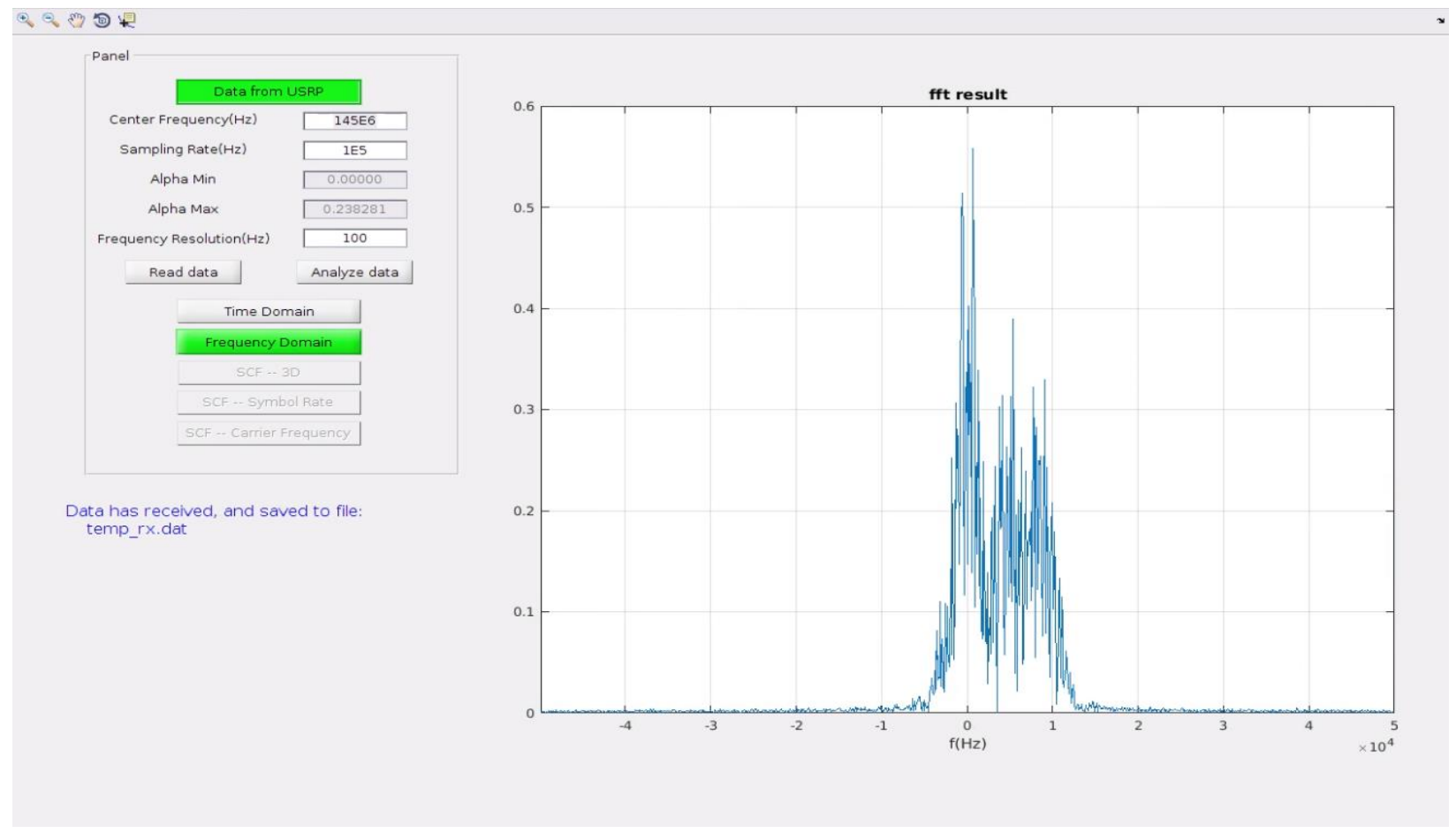

Fig. 7 Frequency domain analysis of signal with significant spectrum overlap

Figure 8 shows the cyclostationary analysis result at the GUI of the SDR based mixed RF signal detector. Specifically, SCF of the down-converted RF signal result is displayed in the window on the right side of the GUI. As seen in Figure 8, the spectrum correlation function is a three dimensional fingerprint of the received RF signal. 


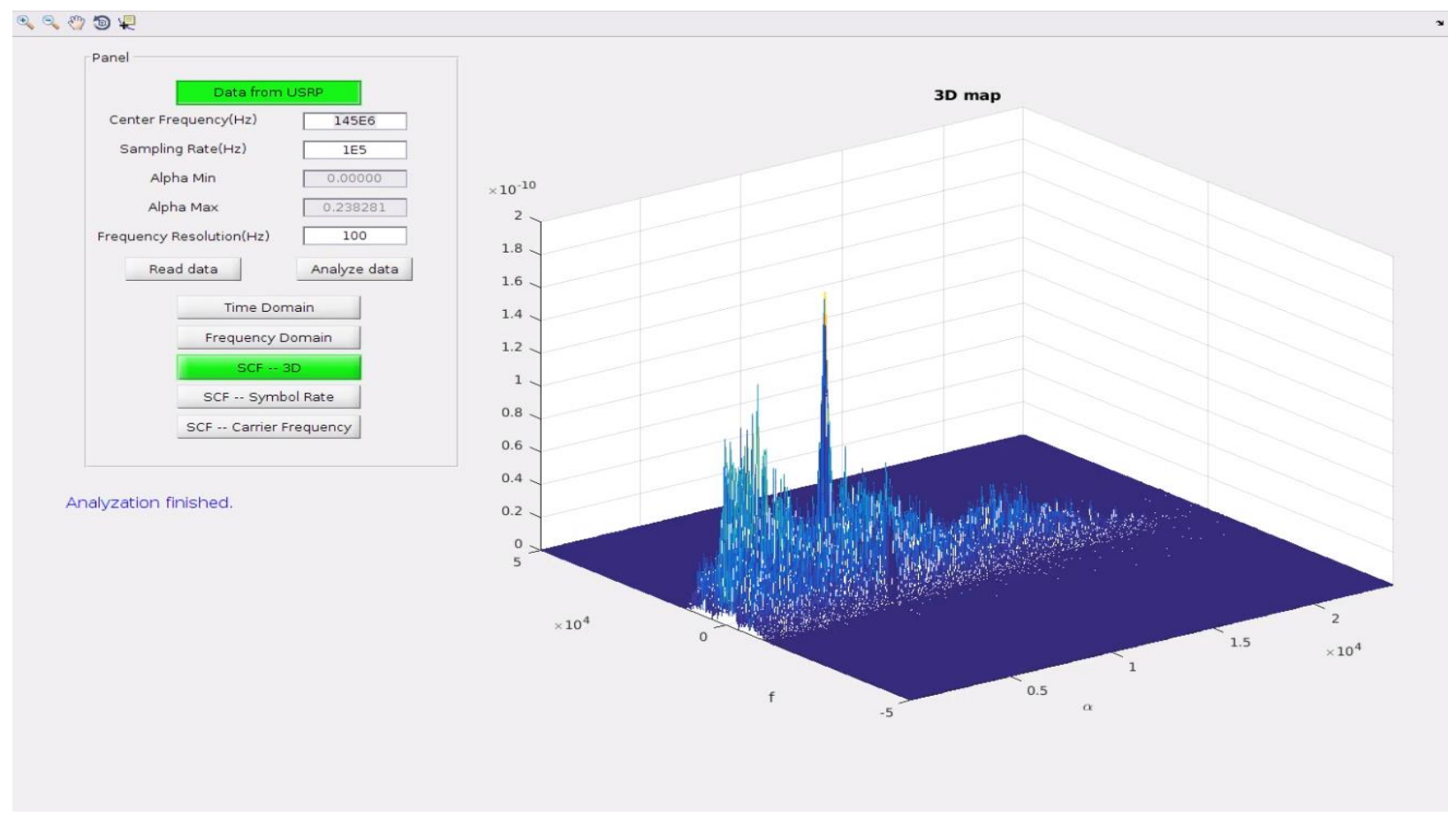

Fig. 8 Cyclostationary analysis of signal with significant spectrum overlap

Figure 9 shows the SCF feature around the symbol rate at the GUI of the SDR based mixed RF signal detector. As seen in Figure 9, the two peaks correspond to the symbol rates of the SCF feature after integration across the frequency domain at a fixed cyclic frequency. It is evident that there are two individual RF signal components in the mixed RF signal even when they are significantly overlapping in spectrum. 


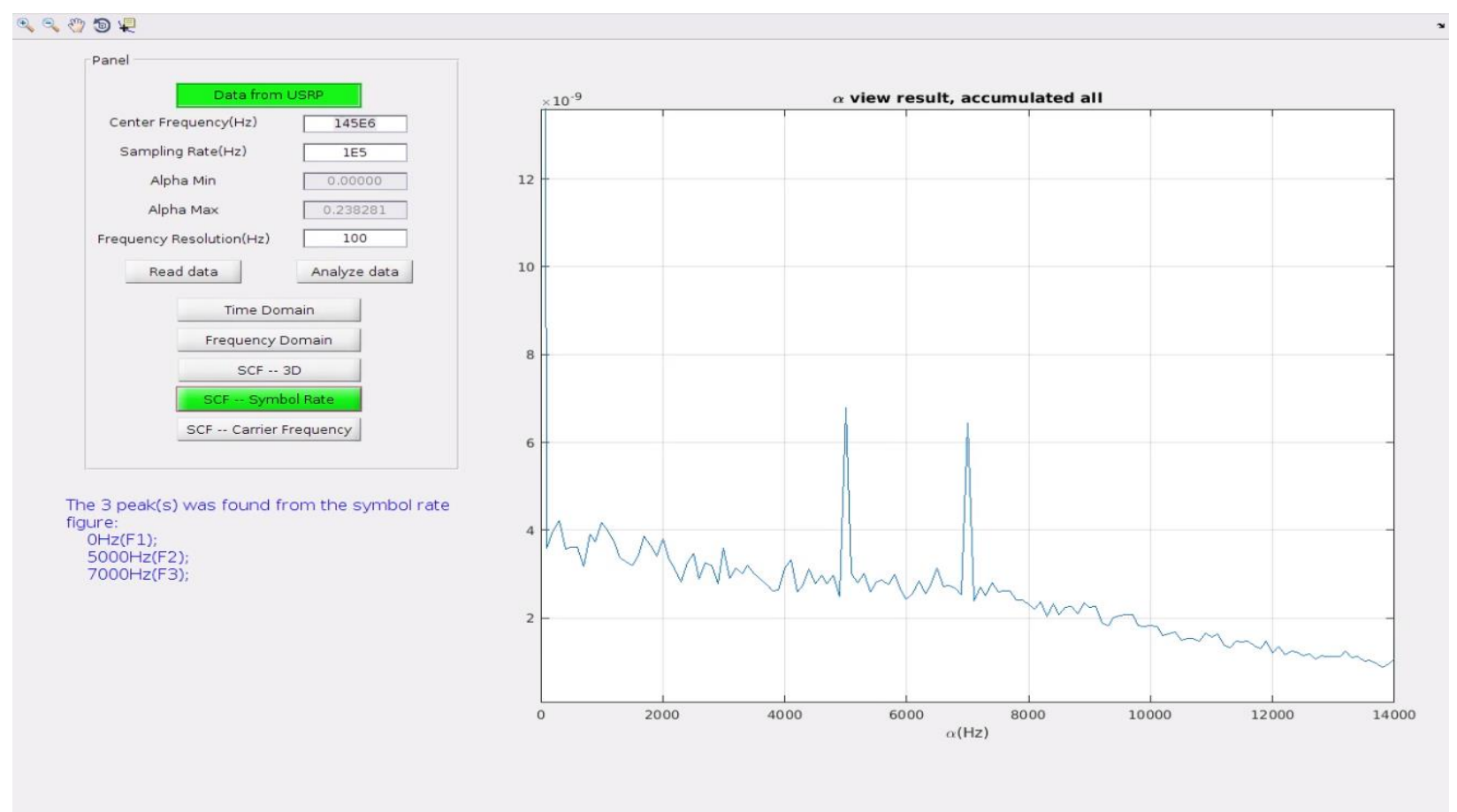

Fig. 9 SCF symbol rate feature of signal with significant spectrum overlap

\section{Enhancing Curricula}

The developed SDR based RF mixed signal detection platform has become a very useful tool to enhance the communication and networking curricula at Wright State University, University of Miami, and Central State University. The platform is very user friendly through its GUI and its graphical display of the signal and analysis results. Labs built upon this platform are being adopted in multiple courses such as EE4210/6210 Digital Communication, EE4730/6730 Wireless Communication, EE7350 Wireless Communication Techniques, and EE8000 Advanced Electronic Warfare.

The platform provides students with an intuitive understanding of the time domain waveform, frequency domain analysis, and cyclostationary analysis of RF signals. By adjusting RF parameters, students are excited and gain important hands-on experiences. During the course review, we have received universal positive feedback on the labs and the SDR based platform.

Due to the graphical manner of the platform, this platform is also a useful tool to attract high school students into STEM fields. We have shown this platform to multiple groups of visiting high school students at Wright State University and invariably students have been highly intrigued by the intuitive graphical display of advanced signal detection and estimation algorithms.

\section{Conclusions}


Supported by an NSF TUES type II grant, we have developed a software defined radio (SDR) based RF mixed signal detection platform for enhancing undergraduate communication and networking curricula. The SDR based platform allows students to observe the communication signals in time domain and frequency domain, as well as cyclostationary analysis domain. Students can easily adjust RF parameters and digital modulation schemes of the communication through a GUI. Additionally, students can perform advanced signal analysis on RF signals. We have developed a series of laboratories on this platform and integrated them in multiple courses across three participating institutions.

\section{References:}

[1] National Instruments Digital Communication Teaching Bundle, http://sine.ni.com/nips/cds/view/p/lang/en/nid/210087

[2] Software Defined Radio Forum, http://www.sdrforum.org

[3] M. Dillinger, K. Madani and N. Alonistioti, Software Defined Radio: Architectures, Systems and Functions, Wiley, 2003

[4] W. Tuttlebee, Software Defined Radio: Enabling Technologies, Wiley, 2002

[5] P. Kenington, $R F$ and Baseband Techniques for Software Defined Radio, Artech House Publishers, 2005

[6] Universal Software Radio Peripheral, http://www.ettus.com/

[7] GNU Radio, http://gnuradio.org

[8] B. Wang, Y. Pei and Z. Wu, "Software Radio Based Wireless Laboratory Design and Implementation for Enhancing Undergraduate Wireless Engineering Education," Proceedings of 2010 Annual ASEE Conference and Exposition, Louisville, KY June 2010.

[9] B. Guenther, B. Rahn, B. Wang and Z. Wu, "Real-Time Video Transmission from High Altitude Balloon: An Interdisciplinary Senior Design Project," Proceedings of 2010 Annual ASEE Conference and Exposition, Louisville, KY June 2010.

[10] C. Cheng, Z. Wu, B. Wang and D. Cao, “'Software Defined Radio for Digital Signal Processing Related Courses," ASEE Annual Conference, 2016

[11] D. Cao, C. Cheng, Z. Wu and B. Wang, "Software Defined Radio Based Laboratories in Undergraduate Computer Networking Courses," ASEE Annual Conference, 2016 
[12] Z. Zhang, Z. Wu, B. Wang, C. Cheng and D. Cao, "Software Defined Radio based General Modulation/Demodulation Platform for Enhancing Undergraduate Communication and Networking Curricula," ASEE Annual Conference, 2016

[13] Z. Zhang, Z. Wu, B. Wang, C. Cheng and D. Cao, “Software Defined Radio based Signal Detection and RF Parameter Estimation Platform for Enhancing Electrical and Computer Engineering Curricula," ASEE Annual Conference, 2016

[14] D. Li, Y. Qu, Z. Liu, Z. Wu and Z. Zhang, "Mixed Signal Detection based on Second-order Cyclostationary Features," IEEE Milcom, 2014

[15] D. Li, J. Ellinger, Z. Liu, Z. Wu and Z. Zhang, "Mixed Signal Detection and Symbol Rate Estimation based on Spectral Coherent Features, "IEEE Milcom 2015

[16] E. C. Like, V. Chakravarthy and Z. Wu, "Signal Classification in Fading Channels Using Cyclic Spectral Analysis," EURASIP Journal on Wireless Communications and Networking, Volume 2009, Article ID 879812

[17] K. Huang, Y. Qu, Z. Zhang, V. Chakarvarthy, L. Zhang and Z. Wu, 'Software Defined Radio based Mixed Signal Detection in Spectrally Congested and Spectrally Contested Environment," NASA 2017 Cognitive Communications for Aerospace Applications Workshop (CCAA) 